\title{
The Interaction of NDF Level and Fiber Particle Size on Productivity and Fiber Digestion in Rabbit during the Fattening Period
}

\author{
M. Sobri ${ }^{a}$, K.G. Wiryawan ${ }^{\text {b, }}$, P.D.M.H. Karti ${ }^{\mathrm{b}}$, \& I.W.T. Wibawan ${ }^{\mathrm{c}}$ \\ ${ }^{a}$ Graduate School of IPB University (Bogor Agricultural University) \\ ${ }^{b}$ Faculty of Animal Science, IPB University (Bogor Agricultural University) \\ Jalan Agatis, Kampus IPB Darmaga, Bogor 16680, Indonesia \\ 'Faculty of Veterinary Medicine, IPB University (Bogor Agricultural University) \\ Jalan Agatis, Kampus IPB Darmaga, Bogor 16680, Indonesia \\ ${ }^{*}$ Corresponding author: kgwiryawan61@gmail.com \\ (Received 10-01-2019; Revised 11-03-2019; Accepted 05-04-2019)
}

\begin{abstract}
This research was conducted to determine the interaction of NDF level and fiber particle size on the performance of production, digestibility of dry matter, crude fiber and fiber fraction, digestive tract, microbial profiles, volatile fatty acids (VFA), and total organic acid of rabbits during the fattening period. The experiment utilized 144 New Zealand white (NZW) crossbred rabbits aged 28 days. The experimental design was a completely randomized $3 \times 3$ factorial pattern, with 3 levels of neutral detergent fiber $(\mathrm{NDF})(\mathrm{F} 1=33.60 \%, \mathrm{~F} 2=36.10 \%$, and $\mathrm{F} 3=41.43 \%)$ and 3 sizes of fiber particles $(\mathrm{P} 1=1 \mathrm{~mm}, \mathrm{P} 2=$ $2 \mathrm{~mm}$, and P3= $3 \mathrm{~mm}$ ). Each treatment had 4 replications, and each replication had 4 animals. The combination treatment showed changes in feed intake with an average of $5.25 \mathrm{~kg} / \mathrm{head}$. The F2 treatment showed the best results in body weight gain (BWG) and feed conversion ratio (FCR) with an average of $1.80 \mathrm{~kg} /$ head and 3.09 , respectively. The F1 level showed the highest average of pathogenic bacteria (E. coli) population at $3.37 \times 109 \mathrm{cfu} / \mathrm{mL}$ which inversely proportional to the average level of butyric acid percentage and total organic acids which were $12.92 \%$ and $386.23 \mathrm{mmol} / \mathrm{L}$ in rabbits during the post-weaning period. The conclusion of this study was that the inclusion of NDF and a balanced size of fiber feed in feed could lead to optimization of the fiber digestibility, digestive tract, total organic acid production, and butyric acid concentration which could suppress the growth of $E$. coli and improve the performance of rabbits during the fattening period.
\end{abstract}

Keywords: NDF; fiber size; fiber digestion; micro flora; rabbits production

\section{INTRODUCTION}

Statistical data reported by Directorate General of PKH (2017) state that rabbit meat contribution is only 554 tons $(0.019 \%)$ of the total 2.98 million ton of national meat production. Rabbits actually have a higher potency than does when it is viewed in terms of productivity and economic aspects, but farmers have found that the mortality rate of rabbits is increasing due to the presence of other diseases and other factors. The NDF level and fiber particle size had significant effects on the cumulative mortality of NZW crossbred rabbits from 5 weeks to 12 weeks of age (fattening period); the average mortality in all treatment groups was $19.44 \%$ (Sobri et al., 2019).

Improving the amount of starch and fiber ratio (with the provision of neutral detergent fiber (NDF) less than $300 \mathrm{~g}$, acid detergent fiber (ADF) less than $150 \mathrm{~g}$, and starch more than $200 \mathrm{~g} / \mathrm{kg}$ ) without changing the cell wall components (eg hemicellulose and lignin) can cause the disruption of feed flow in the intestine, development of the digestive tract, and production of bacterial biomass in the weaning rabbit caecum. The rabbits given too low fiber (less than 8-11 g ADF/kg live weight/day) during the post-weaning period (28-42 days old) and growth period (42-70 days old) will experience a decrease in caecum fiber levels, while if rabbits are given low starch $(15-40 / \mathrm{kg})$ will experience a change in the concentration of fermented products (ammonia and volatile fatty acids (VFA) and caecum pH (De Blas et al., 2012; Gidenne et al., 2013; Marguenda et al., 2012; Villamide et al., 2010). The need for fiber particles increases and is positively correlated with the presence of lignin (Abad et al., 2013; Van Soes et al., 1995; Volek et al., 2011). In general, the particle size of the fiber feed for rabbits is from $0.5-1.5 \mathrm{~mm}$ before pellets are made. The size of the feed particles affects digestion, and it is very important for intestinal motility of rabbits (Abad et al., 2013; Marguenda et al., 2012; Villamide et al., 2010).

Analysis of feed consumption, body weight gain, feed conversion ratio, dry matter and fiber digestibilities, stress status, E. coli, total lactic acid bacteria, acetic acid, propionic acid, butyric acid, and total organic acid in rabbits during the fattening period in this experiment are expected to produce the best treatment combination at standard nutrient requirements for rabbits during 
fattening period. This research is new breakthrough research (potentially produces patent) in examining the problem of rabbit feed in the wet tropics region through a combination of NDF level and fiber particle size.

\section{MATERIALS AND METHODS}

This research was conducted from December 2017 to May 2018 in Pati, Central Java. This study used 144 post-weaning rabbits from 28 days of New Zeland white (NZW). These experimental rabbits were produced through artificial insemination (AI) process of NZW males with 50 local females mated simultaneously using Gonadotropin-releasing hormone $(\mathrm{G} n \mathrm{RH})$ stimulation.

The experimental design was a $3 \times 3$ factorial pattern of completely randomized design, namely 3 levels

Table 1. Nutrients compositions of experimental diet in the rabbit during the fattening period (5-12 weeks)

\begin{tabular}{lcccc}
\hline \multirow{2}{*}{ Nutrient content* } & \multicolumn{3}{c}{ Treatments } & \multirow{2}{*}{ Standard $^{* *}$} \\
\cline { 2 - 4 } & F1 & F2 & F3 & \\
\hline Dry material (\%) & 88.75 & 88.11 & 87.48 & - \\
Crude protein (\%) & 15.00 & 15.02 & 15.03 & $14.20-16.00$ \\
Ash (\%) & 6.19 & 6.49 & 6.79 & - \\
Crude fat (\%) & 6.19 & 6.14 & 6.09 & Free \\
Crude fiber (\%) & 9.75 & 10.83 & 13.89 & $10.00-12.00$ \\
NDF (\%) (F) & 33.60 & 36.10 & 41.43 & $33.00-35.00$ \\
DE (kcal/kg) & 2428 & 2428 & 2428 & 2428 \\
Fiber particle size (P) $(\mathrm{mm})^{* * *}$ & & & $0.5-1.5$ \\
$\quad$ P1 & $33.60 / 1$ & $36.01 / 1$ & $41.43 / 1$ & \\
P2 & $33.60 / 2$ & $36.01 / 2$ & $41.43 / 2$ & \\
P3 & $33.60 / 3$ & $36.01 / 3$ & $41.43 / 3$ & \\
\hline
\end{tabular}

Note: ${ }^{*}$ ) The main ingredients used in treatment feeds were kale straw, rice straw, corn, soybean meal, pollard, palm oil, molasses, and premix based on the analysis at the Laboratory of Animal Science and Technology Faculty of Animal Husbandry Bogor Agricultural University (IPB);

**) Blas \& Wiseman (2010); ***) Marguenda et al. (2012)

$\mathrm{F} 1, \mathrm{~F} 2, \mathrm{~F} 3=3$ levels of NDF ratio; $\mathrm{P} 1, \mathrm{P} 2$, and $\mathrm{P} 3=3$ sizes of fiber particles; $\mathrm{NDF}=$ neutral detergent fiber. of $\mathrm{NDF}$ ratio $(\mathrm{F} 1=33.60 \%, \mathrm{~F} 2=36.10 \%$, and $\mathrm{F} 3=41.43 \%)$ and 3 sizes of fiber particles $(\mathrm{P} 1=1 \mathrm{~mm}, \mathrm{P} 2=2 \mathrm{~mm}$, and $\mathrm{P} 3=3 \mathrm{~mm}$ ) (Table 1). Each treatment was repeated 4 times. Each cage consisted of 4 post-weaning rabbits.

Rice straw and spinach straw were milled to obtain fiber feed with 3 different sizes namely, P1, P2, and P3. The concentrate feed ingredients consisted of corn, polar, soybean meal, palm oil, molasses, and premix. The raw ingredients for animal feed that were used as a treatment ration or after being completed feed were analyzed in advance for its nutritional content. Proximate test using the AOAC method (2012) and Fiber Fraction Test using the Van Soest method (1995) were carried out on feed raw materials and complete feed.

The variables observed were body weight gain (BWG), feed conversion ratio (FCR), dry matter (DM) and fiber digestibilities, stress status, E. coli, total lactic acid bacteria (LAB), acetic acid, propionic acid, butyric acid, and total organic acid (TOA) in the contents of the caecum.

The collections of the caecum and digestive tract organ samples were carried out 2 times, namely at the age of 42 days (post-weaning period) and 70 days (growth period). All actions taken in this study were approved by the Animal Ethics Commission, Bogor Agricultural University with ethical approval numbers: 86-2017 IPB dated December 19, 2017. Data for each observed variable were analyzed by variance, while the mean difference was tested by Duncan's multiple distances (Steel \& Torrie, 1995).

\section{RESULTS}

The overall temperature inside the room during the experiment was $26.01{ }^{\circ} \mathrm{C}$ (close house) while the range of outside temperature was $23.9-32.2{ }^{\circ} \mathrm{C}$, and the range of humidity was between $70.66 \%-97.66 \%$ with an average of $86.51 \%$. The levels of NDF and fiber particle size gave a significant interaction effect $(\mathrm{p}<0.05)$ on the $\mathrm{cu}-$ mulative consumption of NZW crossbred rabbits from

Table 2. The effect of NDF (F) level and fiber particle size (P) on FI, BWG, and FCR of New Zeland white crossbred rabbits during the fattening period (5-12 weeks)

\begin{tabular}{|c|c|c|c|c|c|}
\hline \multirow{2}{*}{ Variables } & \multirow{2}{*}{$\begin{array}{c}\text { Fiber particle } \\
\text { size }\end{array}$} & \multicolumn{3}{|c|}{ Treatments } & \multirow{2}{*}{ Average } \\
\hline & & F1 & $\mathrm{F} 2$ & F3 & \\
\hline \multirow[t]{3}{*}{$\mathrm{FI}(\mathrm{kg})^{*}$} & P1 & $5.23 \pm 0.60 q$ & $5.28 \pm 0.60 \mathrm{q}$ & $4.36 \pm 0.50 q$ & $4.96 \pm 0.57$ \\
\hline & P2 & $5.60 \pm 0.64 p$ & $5.05 \pm 0.58 \mathrm{q}$ & $5.60 \pm 0.64 p$ & $5.42 \pm 0.62$ \\
\hline & P3 & $4.59 \pm 0.52 q$ & $6.00 \pm 0.68 p$ & $5.51 \pm 0.63 p$ & $5.36 \pm 0.61$ \\
\hline \multirow[t]{2}{*}{ Average } & & $5.14 \pm 0.08$ & $5.44 \pm 0.09$ & $5.16 \pm 0.08$ & $5.25 \pm 0.08$ \\
\hline & P1 & $1.33 \pm 0.22$ & $1.87 \pm 0.31$ & $1.66 \pm 0.28$ & $1.62 \pm 0.27$ \\
\hline \multirow[t]{2}{*}{ Body weight gain/BWG (kg) } & P2 & $1.83 \pm 0.31$ & $1.66 \pm 0.28$ & $1.73 \pm 0.29$ & $1.74 \pm 0.29$ \\
\hline & P3 & $1.22 \pm 0.21$ & $1.89 \pm 0.32$ & $1.63 \pm 0.27$ & $1.58 \pm 0.27$ \\
\hline \multirow[t]{2}{*}{ Average ${ }^{* *}$} & & $1.46 \pm 0.02^{\mathrm{a}}$ & $1.80 \pm 0.03^{b}$ & $1.67 \pm 0.03^{b}$ & $1.64 \pm 0.28$ \\
\hline & P1 & $4.07 \pm 0.76$ & $2.97 \pm 0.55$ & $2.66 \pm 0.49$ & $3.23 \pm 0.60$ \\
\hline \multirow[t]{2}{*}{ Feed consumption ratio (FCR) } & $\mathrm{P} 2$ & $3.05 \pm 0.57$ & $3.10 \pm 0.58$ & $3.23 \pm 0.60$ & $3.13 \pm 0.58$ \\
\hline & P3 & $3.86 \pm 0.72$ & $3.20 \pm 0.59$ & $3.47 \pm 0.64$ & $3.51 \pm 0.65$ \\
\hline Average ** & & $3.66 \pm 0.68^{a}$ & $3.09 \pm 0.57^{b}$ & $3.12 \pm 0.58^{b}$ & $3.29 \pm 0.61$ \\
\hline
\end{tabular}

Note: ${ }^{*}$ ) Means in the same combination row and column with different superscripts differ significantly $(p<0.05)$.

**) Means in the same row with different superscripts differ significantly $(p<0.05)$.

F1, F2, and F3= 3 levels of NDF ratio; P1, P2, and P3= 3 sizes of fiber particles. 
5 weeks to 12 weeks (fattening period) with an average of $5.25 \mathrm{~kg} /$ head (Table 2). The difference in NDF level showed the effect $(\mathrm{p}<0.05)$ on body weight gain $(B W G)$ and feed consumption ratio (FCR). NDF at the F2 level showed the best results on all BWG averages of $1.80 \mathrm{~kg} /$ head with an FCR of 3.09 (Table 2).

Different levels of NDF content and fiber particle size gave a significant interaction effect $(p<0.05)$ on dry matter digestibility during the post-weaning period (5 weeks to 6 weeks) with an average of $63.23 \%$ (Table 3 ). The effect of different NDF concentrations and fiber particle sizes gave a significant interaction effect $(\mathrm{p}<0.05)$ on the digestibility of crude fiber during the growth period ( 7 weeks to 10 weeks) with an average value of all combinations of $33.17 \%$ (Table 4 ). The lowest level of NDF (F1) showed the effect $(p<0.05)$ on crude fiber digestibility with the highest crude fiber digestibility (48.95\%) during the post-weaning period (Table 3).

Different NDF contents and fiber particle sizes during the post-weaning period and growth period gave significant interaction effects $(p<0.05)$ on the digestibilities of NDF, ADF, and cellulose. Each treatment com-

Table 3. The nutrient digestibility, caecum length/life weight (CL/LW), E. coli (cfu/mL) and total lactic acid bacteria (LAB) of of New Zeland white crossbred rabbits during the postweaning periods (42 days) treated by different level of NDF (F) and fiber particle size $(P)$

\begin{tabular}{|c|c|c|c|c|c|}
\hline \multirow{2}{*}{ Variables } & \multirow{2}{*}{$\begin{array}{l}\text { Fiber particle } \\
\text { size }\end{array}$} & \multicolumn{3}{|c|}{ Treatments } & \multirow{2}{*}{ Average } \\
\hline & & F1 & F2 & F3 & \\
\hline \multirow[t]{3}{*}{ Dry matter digestibility (\%) * } & P1 & $68.42 \pm 1.10 p$ & $60.01 \pm 0.97^{s}$ & $58.16 \pm 0.94^{\mathrm{t}}$ & $62.20 \pm 1.00$ \\
\hline & $\mathrm{P} 2$ & $66.27 \pm 1.07 q$ & $61.39 \pm 0.99^{\mathrm{rs}}$ & $64.94 \pm 1.05^{\mathrm{q}}$ & $64.20 \pm 1.03$ \\
\hline & P3 & $69.35 \pm 1.12^{p}$ & $62.40 \pm 1.00^{r}$ & $58.11 \pm 0.94^{\mathrm{t}}$ & $63.29 \pm 1.02$ \\
\hline Average & & $68.01 \pm 1.10$ & $61.27 \pm 0.99$ & $60.40 \pm 0.97$ & $63.23 \pm 1.02$ \\
\hline \multirow[t]{3}{*}{ Crude fiber digestibility (\%) } & P1 & $49.36 \pm 6.69$ & $35.76 \pm 4.85$ & $39.48 \pm 5.35$ & $41.53 \pm 5.63$ \\
\hline & $\mathrm{P} 2$ & $46.64 \pm 6.32$ & $36.64 \pm 4.96$ & $42.29 \pm 5.73$ & $41.86 \pm 5.67$ \\
\hline & P3 & $50.85 \pm 6.89$ & $38.87 \pm 5.27$ & $30.55 \pm 4.14$ & $40.09 \pm 5.43$ \\
\hline Average $^{* *}$ & & $48.95 \pm 6.63^{a}$ & $37.09 \pm 5.03^{\mathrm{b}}$ & $37.44 \pm 5.07 \mathrm{~b}$ & $41.16 \pm 5.58$ \\
\hline \multirow[t]{3}{*}{ NDF digestibility (\%) * } & P1 & $53.51 \pm 1.36 \mathrm{p}$ & $48.64 \pm 1.24^{\mathrm{qr}}$ & $49.91 \pm 1.27 \mathrm{q}$ & $50.69 \pm 1.29$ \\
\hline & $\mathrm{P} 2$ & $48.47 \pm 1.23^{q \mathrm{r}}$ & $40.59 \pm 1.03^{\mathrm{s}}$ & $54.75 \pm 1.39 p$ & $47.94 \pm 1.22$ \\
\hline & P3 & $54.56 \pm 1.39 p$ & $53.25 \pm 1.35 \mathrm{p}$ & $45.59 \pm 1.16^{\mathrm{r}}$ & $51.13 \pm 1.30$ \\
\hline Average & & $52.18 \pm 1.33$ & $47.50 \pm 1.21$ & $50.08 \pm 1.27$ & $49.92 \pm 1.27$ \\
\hline \multirow[t]{3}{*}{ ADF digestibility (\%)* } & P1 & $28.80 \pm 1.81^{\mathrm{s}}$ & $19.15 \pm 1.20^{\mathrm{t}}$ & $38.42 \pm 2.42 \mathrm{p}$ & $28.79 \pm 1.81$ \\
\hline & $\mathrm{P} 2$ & $28.43 \pm 1.79 \mathrm{~s}$ & $18.71 \pm 1.18^{t}$ & $35.60 \pm 2.24^{\mathrm{pq}}$ & $27.58 \pm 1.73$ \\
\hline & P3 & $30.60 \pm 1.92^{\mathrm{rs}}$ & $33.00 \pm 2.08^{\mathrm{qr}}$ & $32.57 \pm 2.05^{\mathrm{qr}}$ & $32.05 \pm 2.02$ \\
\hline Average & & $29.28 \pm 1.84$ & $23.62 \pm 1.49$ & $35.53 \pm 2.23$ & $29.48 \pm 1.85$ \\
\hline \multirow[t]{3}{*}{ Cellulose digestibility $(\%){ }^{*}$} & P1 & $18.21 \pm 2.24^{\mathrm{r}}$ & $18.71 \pm 2.30^{\mathrm{r}}$ & $21.93 \pm 2.69 q$ & $19.62 \pm 2.41$ \\
\hline & $\mathrm{P} 2$ & $17.80 \pm 2.19^{\mathrm{r}}$ & $23.50 \pm 2.89 q$ & $16.06 \pm 1.97^{r}$ & $19.12 \pm 2.35$ \\
\hline & P3 & $28.16 \pm 3.46^{p}$ & $6.51 \pm 0.80^{\mathrm{s}}$ & $2.52 \pm 0.31^{\mathrm{t}}$ & $12.39 \pm 1.52$ \\
\hline Average & & $21.39 \pm 2.63$ & $16.24 \pm 1.99$ & $13.50 \pm 1.66$ & $17.04 \pm 2.09$ \\
\hline \multirow[t]{3}{*}{ Lignin digestibility (\%) } & $\mathrm{P} 1$ & $12.93 \pm 1.93$ & $8.03 \pm 1.20$ & $21.74 \pm 3.25$ & $14.23 \pm 2.13$ \\
\hline & $\mathrm{P} 2$ & $12.90 \pm 1.93$ & $7.96 \pm 1.19$ & $20.34 \pm 3.04$ & $13.73 \pm 2.05$ \\
\hline & P3 & $12.97 \pm 1.94$ & $7.94 \pm 1.19$ & $20.32 \pm 3.04$ & $13.75 \pm 2.05$ \\
\hline \multirow[t]{2}{*}{ Average $^{* *}$} & & $12.94 \pm 1.93^{\mathrm{b}}$ & $7.98 \pm 1.19 c$ & $20.80 \pm 3.11^{\mathrm{a}}$ & $13.90 \pm 2.08$ \\
\hline & $\mathrm{P} 1$ & $0.046 \pm 0.01^{p q}$ & $0.027 \pm 0.01^{\mathrm{r}}$ & $0.055 \pm 0.01^{p}$ & $0.043 \pm 0.01$ \\
\hline \multirow[t]{2}{*}{$\mathrm{CL} / \mathrm{LW}^{*}$} & $\mathrm{P} 2$ & $0.052 \pm 0.01^{\mathrm{p}}$ & $0.041 \pm 0.01^{\text {pqr }}$ & $0.032 \pm 0.01^{\mathrm{qr}}$ & $0.042 \pm 0.01$ \\
\hline & P3 & $0.058 \pm 0.01^{p}$ & $0.050 \pm 0.01^{\mathrm{p}}$ & $0.044 \pm 0.01^{\mathrm{pq}}$ & $0.051 \pm 0.01$ \\
\hline Average & & $0.052 \pm 0.01$ & $0.039 \pm 0.01$ & $0.044 \pm 0.01$ & $0.045 \pm 0.01$ \\
\hline \multirow[t]{3}{*}{ E. coli $10^{9}(\mathrm{cfu} / \mathrm{mL})$} & P1 & $3.47 \pm 0.46$ & $2.74 \pm 0.36$ & $2.98 \pm 0.39$ & $3.06 \pm 0.41^{a}$ \\
\hline & $\mathrm{P} 2$ & $2.84 \pm 0.38$ & $2.71 \pm 0.36$ & $2.55 \pm 0.34$ & $2.70 \pm 0.36^{\mathrm{b}}$ \\
\hline & P3 & $3.80 \pm 0.50$ & $3.41 \pm 0.45$ & $2.64 \pm 0.35$ & $3.28 \pm 0.43^{a}$ \\
\hline Average $^{* *}$ & & $3.37 \pm 0.45^{\mathrm{a}}$ & $2.95 \pm 0.39^{b}$ & $2.72 \pm 0.36^{b}$ & $3.01 \pm 0.40$ \\
\hline \multirow[t]{3}{*}{$\mathrm{LAB} 10^{9}(\mathrm{cfu} / \mathrm{mL})^{*}$} & $\mathrm{P} 1$ & $3.03 \pm 0.55^{\mathrm{pqr}}$ & $2.47 \pm 0.45^{\mathrm{qrs}}$ & $3.00 \pm 0.54^{\mathrm{pqr}}$ & $2.84 \pm 0.51$ \\
\hline & $\mathrm{P} 2$ & $1.77 \pm 0.32^{\mathrm{s}}$ & $3.35 \pm 0.60 p$ & $1.82 \pm 0.33^{s}$ & $2.31 \pm 0.42$ \\
\hline & P3 & $2.40 \pm 0.43^{\mathrm{qrs}}$ & $3.14 \pm 0.57 \mathrm{pq}$ & $2.30 \pm 0.42^{\mathrm{rs}}$ & $2.61 \pm 0.47$ \\
\hline Average & & $2.40 \pm 0.43$ & $2.99 \pm 0.54$ & $2.37 \pm 0.43$ & $2.59 \pm 0.47$ \\
\hline
\end{tabular}

Note: $\left.{ }^{*}\right)$ Means in the same combination row and column with different superscripts differ significantly $(p<0.05)$.

**) Means in the same row with different superscripts differ significantly $(p<0.05)$.

***) Means in the same column with different superscripts differ significantly $(\mathrm{p}<0.05)$

F1, F2, and F3= 3 levels of NDF ratio; P1, P2, and P3= 3 sizes of fiber particles; $\mathrm{NDF}=$ neutral detergent fiber; $\mathrm{ADF}=$ acid detergent fiber. 
bination gave average digestibilities of $49.92 \%, 29.48 \%$, and $17.04 \%$, respectively, during the post-weaning period (Table 3 ) and $50.86 \%, 27.63 \%$, and $20.87 \%$, respectively, during the fattening period (Table 4). Giving the high levels of NDF had a significant effect $(p<0.05)$ on the digestibility of lignin. The mean value of the highest lignin digestibility during the post-weaning period was found in group F1, which was $20.80 \%$ with the average of all treatments was $13.90 \%$ (Table 3 ). While during the fattening period, the F1 group showed a lignin digestibility value of $21.45 \%$ with an average of all treatments was $14.14 \%$ (Table 4 ).
The differences in NDF concentration and fiber particle size during the fattening period had a significant effect $(p<0.05)$ on the length of caecum per live weight. Interaction effects of F1P3 and F3P1 groups during the post-weaning period showed the highest mean values at caecum length per live weight i.e., 0.058 and 0.055 , respectively (Table 3). Meanwhile, during the growth period, the F1P1 and F3P2 groups showed the highest scores on the average length of caecum per live weight i.e., 0.080 and 0.046 (Table 4).

NDF level and fiber particle size gave a significant interaction effect $(p<0.05)$ on the number of $E$. coli bacte-

Table 4. The nutrient digestibility, caecum length/life weight (CL/LW), E. coli (cfu/mL), and total lactic acid bacteria (LAB) of of New Zeland white crossbred rabbits during the growth periods (70 days) treated by different level of NDF (F) and fiber particle size $(\mathrm{P})$

\begin{tabular}{|c|c|c|c|c|c|}
\hline \multirow{2}{*}{ Variables } & \multirow{2}{*}{$\begin{array}{c}\text { Fiber particle } \\
\text { size }\end{array}$} & \multicolumn{3}{|c|}{ Treatments } & \multirow{2}{*}{ Average } \\
\hline & & F1 & F2 & F3 & \\
\hline \multirow[t]{3}{*}{ Dry matter digestibility (\%)* } & P1 & $69.44 \pm 1.01^{\mathrm{p}}$ & $61.77 \pm 0.90^{\mathrm{rt}}$ & $63.05 \pm 0.91 q^{\mathrm{qr}}$ & $64.75 \pm 0.94$ \\
\hline & P2 & $68.75 \pm 1.00^{\mathrm{p}}$ & $60.72 \pm 0.88^{\text {tu }}$ & $59.79 \pm 0.87^{u}$ & $63.09 \pm 0.91$ \\
\hline & P3 & $69.13 \pm 1.00^{\mathrm{p}}$ & $60.56 \pm 0.88^{\text {tu }}$ & $64.06 \pm 0.93^{q}$ & $64.58 \pm 0.94$ \\
\hline Average & & $69.11 \pm 1.00$ & $61.02 \pm 0.88$ & $62.30 \pm 0.90$ & $64.14 \pm 0.93$ \\
\hline \multirow[t]{3}{*}{ Crude fiber digestibility (\%) } & P1 & $37.66 \pm 3.99 \mathrm{pq}$ & $23.01 \pm 2.44^{\mathrm{s}}$ & $35.98 \pm 3.81^{q}$ & $32.22 \pm 3.41$ \\
\hline & P2 & $37.80 \pm 4.00^{\mathrm{pq}}$ & $27.82 \pm 2.95^{\mathrm{rs}}$ & $30.27 \pm 3.21^{\mathrm{r}}$ & $31.96 \pm 3.38$ \\
\hline & P3 & $36.56 \pm 3.87^{q}$ & $26.95 \pm 2.85^{\mathrm{rs}}$ & $42.50 \pm 4.50^{\mathrm{p}}$ & $35.34 \pm 3.74$ \\
\hline Average ${ }^{* *}$ & & $37.34 \pm 3.95$ & $25.93 \pm 2.75$ & $36.25 \pm 3.84$ & $33.17 \pm 3.51$ \\
\hline \multirow[t]{3}{*}{ NDF digestibility (\%) * } & P1 & $58.02 \pm 1.47^{p}$ & $39.80 \pm 1.01^{\mathrm{r}}$ & $51.29 \pm 1.30^{q}$ & $49.70 \pm 1.26$ \\
\hline & P2 & $50.04 \pm 1.27^{q}$ & $50.10 \pm 1.27^{q}$ & $49.71 \pm 1.26^{q}$ & $49.95 \pm 1.26$ \\
\hline & P3 & $56.86 \pm 1.44^{\mathrm{p}}$ & $50.45 \pm 1.28^{q}$ & $51.50 \pm 1.30^{q}$ & $52.94 \pm 1.34$ \\
\hline Average & & $54.97 \pm 1.39$ & $46.78 \pm 1.18$ & $50.83 \pm 1.29$ & $50.86 \pm 1.29$ \\
\hline \multirow[t]{3}{*}{ ADF digestibility (\%)* } & P1 & $36.63 \pm 2.87^{\mathrm{pq}}$ & $7.03 \pm 0.55^{\mathrm{s}}$ & $37.31 \pm 2.92^{\mathrm{pq}}$ & $26.99 \pm 2.11$ \\
\hline & P2 & $34.44 \pm 2.70^{q}$ & $10.52 \pm 0.82^{\mathrm{s}}$ & $32.24 \pm 2.52^{q}$ & $25.73 \pm 2.01$ \\
\hline & P3 & $34.57 \pm 2.71^{\mathrm{pq}}$ & $17.51 \pm 1.37^{\mathrm{r}}$ & $38.40 \pm 3.01^{\mathrm{p}}$ & $30.16 \pm 2.36$ \\
\hline Average & & $35.21 \pm 2.76$ & $11.69 \pm 0.92$ & $35.98 \pm 2.82$ & $27.63 \pm 2.16$ \\
\hline \multirow[t]{3}{*}{ Cellulose digestibility (\%)* } & P1 & $30.26 \pm 3.43^{p}$ & $21.56 \pm 2.45^{\mathrm{qr}}$ & $6.89 \pm 0.78^{\mathrm{u}}$ & $19.57 \pm 2.22$ \\
\hline & P2 & $24.35 \pm 2.76^{\mathrm{q}}$ & $19.03 \pm 2.16^{\mathrm{r}}$ & $11.33 \pm 1.29^{\mathrm{t}}$ & $18.24 \pm 2.07$ \\
\hline & P3 & $30.56 \pm 3.47^{p}$ & $20.64 \pm 2.34^{\mathrm{r}}$ & $11.42 \pm 1.30^{\mathrm{t}}$ & $20.87 \pm 2.37$ \\
\hline Average & & $28.39 \pm 3.22$ & $20.41 \pm 2.32$ & $9.88 \pm 1.12$ & $19.56 \pm 2.22$ \\
\hline \multirow[t]{3}{*}{ Lignin digestibility (\%) } & P1 & $12.97 \pm 1.81$ & $7.98 \pm 1.11$ & $22.38 \pm 3.12$ & $14.44 \pm 2.02$ \\
\hline & P2 & $13.12 \pm 1.83$ & $8.02 \pm 1.12$ & $20.93 \pm 2.92$ & $14.02 \pm 1.96$ \\
\hline & P3 & $12.98 \pm 1.81$ & $8.02 \pm 1.12$ & $21.04 \pm 2.94$ & $14.01 \pm 1.96$ \\
\hline \multirow[t]{2}{*}{ Average** } & & $13.02 \pm 1.82^{\mathrm{b}}$ & $8.00 \pm 1.12^{c}$ & $21.45 \pm 2.99^{a}$ & $14.16 \pm 1.98$ \\
\hline & P1 & $0.080 \pm 0.019^{p}$ & $0.028 \pm 0.007^{\mathrm{r}}$ & $0.037 \pm 0.009^{q}$ & $0.048 \pm 0.011$ \\
\hline \multirow[t]{2}{*}{$\mathrm{CL} / \mathrm{LW}^{*}$} & P2 & $0.030 \pm 0.007^{\mathrm{r}}$ & $0.029 \pm 0.007^{r}$ & $0.046 \pm 0.011^{\mathrm{q}}$ & $0.035 \pm 0.008$ \\
\hline & P3 & $0.038 \pm 0.009^{q}$ & $0.027 \pm 0.006^{\mathrm{r}}$ & $0.030 \pm 0.007^{\mathrm{r}}$ & $0.032 \pm 0.008$ \\
\hline Average & & $0.050 \pm 0.012$ & $0.028 \pm 0.007$ & $0.038 \pm 0.009$ & $0.038 \pm 0.009$ \\
\hline \multirow[t]{3}{*}{ E. coli $10^{9}(\mathrm{cfu} / \mathrm{mL})^{*}$} & P1 & $2.40 \pm 0.53^{\mathrm{rs}}$ & $3.31 \pm 0.74^{\mathrm{pqs}}$ & $4.04 \pm 0.90^{\mathrm{p}}$ & $3.25 \pm 0.72$ \\
\hline & P2 & $2.90 \pm 0.64^{\mathrm{pqrs}}$ & $3.31 \pm 0.74^{\mathrm{pqr}}$ & $2.82 \pm 0.63^{\mathrm{qrs}}$ & $3.01 \pm 0.67$ \\
\hline & P3 & $3.74 \pm 0.83^{\mathrm{pq}}$ & $2.94 \pm 0.65^{\mathrm{pqr}}$ & $2.27 \pm 0.50^{\mathrm{rs}}$ & $2.98 \pm 0.66$ \\
\hline Average & & $3.01 \pm 0.67$ & $3.19 \pm 0.71$ & $3.04 \pm 0.68$ & $3.08 \pm 0.68$ \\
\hline \multirow[t]{3}{*}{ LAB $10^{9}(\mathrm{cfu} / \mathrm{mL})^{*}$} & P1 & $2.77 \pm 0.33^{\mathrm{pqr}}$ & $2.59 \pm 0.31^{\mathrm{pqr}}$ & $2.74 \pm 0.33^{\text {pqr }}$ & $2.70 \pm 0.32$ \\
\hline & P2 & $2.54 \pm 0.30^{\mathrm{qr}}$ & $3.11 \pm 0.37^{p}$ & $1.98 \pm 0.24^{\mathrm{r}}$ & $2.55 \pm 0.31$ \\
\hline & P3 & $2.95 \pm 0.35^{\mathrm{pq}}$ & $2.82 \pm 0.34^{\mathrm{pq}}$ & $2.59 \pm 0.31^{\mathrm{pqr}}$ & $2.79 \pm 0.33$ \\
\hline Average & & $2.75 \pm 0.33$ & $2.84 \pm 0.34$ & $2.44 \pm 0.29$ & $2.68 \pm 0.32$ \\
\hline
\end{tabular}

Note: ${ }^{*}$ ) Means in the same combination row and column with different superscripts differ significantly $(\mathrm{p}<0.05)$.

$\left.{ }^{* *}\right)$ Means in the same row with different superscripts differ significantly $(p<0.05)$.

$\mathrm{F} 1, \mathrm{~F} 2$, and F3= 3 levels of NDF ratio; $\mathrm{P} 1, \mathrm{P} 2$, and $\mathrm{P} 3=3$ sizes of fiber particles; $\mathrm{NDF}=$ neutral detergent fiber; $\mathrm{ADF}=$ acid detergent fiber. 
ria in the contents of the caecum with an average of 3.08 x $10^{9} \mathrm{cfu} / \mathrm{mL}$ during the growth period (Table 4 ). The highest average population of E. coli in the contents of the caecum was found in the rabbits treated at the level of NDF F1 $(p<0.05)$, which was $3.37 \times 10^{9} \mathrm{cfu} / \mathrm{mL}$ and fiber particle size of P1 and P3 $(\mathrm{p}<0.05)$, which were $3.06 \mathrm{x}$ $10^{9} \mathrm{cfu} / \mathrm{mL}$ and $3.28 \times 10^{9} \mathrm{cfu} / \mathrm{mL}$ in NZW crossbred rabbits during the post-weaning period (Table 3). The F3P2 group showed the highest total population of lactic acid bacteria (618.65 mmol/L) (Table 5) and the lowest E. coli population $\left(2.55 \times 10^{9} \mathrm{cfu} / \mathrm{mL}\right)$ (Table 3$)$ during the postweaning periods. The best fiber particle size at the level of P2 could suppress the growth of E. coli bacteria on the contents of the caecum with the highest average or the lowest population rate of $2.70 \times 10^{9} \mathrm{cfu} / \mathrm{mL}$ during the post-weaning period (Table 3 ).

The treatments of different NDF contents and fiber particle sizes gave a significant interaction effect $(p<0.05)$ on the total content of lactic acid bacteria in the caecum contents both during the post-weaning period $\left(2.59 \times 10^{9}\right.$ $\mathrm{cfu} / \mathrm{mL})$ (Table 3$)$ and during the growth period $(2.68 \mathrm{x}$ $10^{9} \mathrm{cfu} / \mathrm{mL}$ ) (Table 4) in NZW crossbred rabbits.

The differences in NDF contents and fiber particle sizes gave significant interaction effects $(p<0.05)$ on the percentage of acetic acid and propionic acid in the caecum contents during the post-weaning period with a mean of $78.98 \%$ and $8.24 \%$, respectively (Table 5). Meanwhile, during the growth period, the percentages

Table 5. Proportional VFA (acetic acid, propionic acid and butyric acid) and total organic acid (TOA) in the contents caecum of NZW crossbred rabbits during the postweaning periods (42 days) and growth periods (70 days) treated by different level of NDF (F) and fiber particle size $(\mathrm{P})$

\begin{tabular}{|c|c|c|c|c|c|}
\hline \multirow{2}{*}{ Variables } & \multirow{2}{*}{$\begin{array}{l}\text { Fiber particle } \\
\text { size }\end{array}$} & \multicolumn{3}{|c|}{ Treatments } & \multirow{2}{*}{ Average ${ }^{* *}$} \\
\hline & & F1 & F2 & F3 & \\
\hline \multicolumn{6}{|c|}{ Postweaning period ( 42 days) } \\
\hline \multirow[t]{3}{*}{ Acetic acid $(\%)^{*}$} & P1 & $72.82 \pm 2.36^{r}$ & $75.32 \pm 2.44^{\mathrm{r}}$ & $77.85 \pm 2.52^{q}$ & $75.33 \pm 2.44$ \\
\hline & $\mathrm{P} 2$ & $87.79 \pm 2.84 \mathrm{p}$ & $77.97 \pm 2.53 q$ & $81.67 \pm 2.65 q$ & $82.48 \pm 2.67$ \\
\hline & P3 & $80.74 \pm 2.62 q$ & $80.53 \pm 2.61^{q}$ & $75.86 \pm 2.46^{\mathrm{r}}$ & $79.04 \pm 2.56$ \\
\hline Average & & $80.45 \pm 2.61$ & $77.94 \pm 2.53$ & $78.46 \pm 2.54$ & $78.95 \pm 2.56$ \\
\hline \multirow{3}{*}{ Propionic acid $(\%)^{*}$} & $\mathrm{P} 1$ & $9.91 \pm 1.38 \mathrm{p}$ & $6.88 \pm 0.96^{\mathrm{rs}}$ & $8.36 \pm 1.17 \mathrm{pqr}$ & $8.38 \pm 1.17$ \\
\hline & P2 & $5.73 \pm 0.80^{\mathrm{s}}$ & $7.40 \pm 1.03^{\mathrm{qr}}$ & $8.96 \pm 1.25^{\mathrm{pq}}$ & $7.37 \pm 1.03$ \\
\hline & P3 & $7.73 \pm 1.08^{\mathrm{qr}}$ & $9.37 \pm 1.31^{\mathrm{pq}}$ & $9.81 \pm 1.37 \mathrm{p}$ & $8.97 \pm 1.25$ \\
\hline Average & & $7.79 \pm 1.09$ & $7.88 \pm 1.10$ & $9.04 \pm 1.26$ & $8.24 \pm 1.15$ \\
\hline \multirow[t]{3}{*}{ Butyric acid (\%) } & P1 & $17.08 \pm 0.43$ & $22.77 \pm 0.58$ & $18.21 \pm 0.46$ & $19.35 \pm 0.49^{a}$ \\
\hline & P2 & $7.45 \pm 0.19$ & $15.63 \pm 0.40$ & $11.39 \pm 0.29$ & $11.49 \pm 0.29^{\mathrm{b}}$ \\
\hline & P3 & $14.23 \pm 0.36$ & $12.19 \pm 0.31$ & $17.03 \pm 0.43$ & $14.48 \pm 0.37 \mathrm{~b}$ \\
\hline Average & & $12.92 \pm 0.33$ & $16.86 \pm 0.43$ & $15.54 \pm 0.39$ & $15.11 \pm 0.38$ \\
\hline \multirow[t]{3}{*}{$\mathrm{TOA}(\mathrm{mmol} / \mathrm{L})^{*}$} & P1 & $335.89 \pm 70.13^{\text {st }}$ & $435.56 \pm 90.95^{\text {qrst }}$ & $369.89 \pm 77.23 p$ & $380.45 \pm 79.44$ \\
\hline & P2 & $333.18 \pm 69.57^{\mathrm{t}}$ & $430.90 \pm 89.97^{\text {qrst }}$ & $618.65 \pm 129.17^{\mathrm{pq}}$ & $460.91 \pm 96.24$ \\
\hline & P3 & $489.60 \pm 102.23^{\mathrm{pr}}$ & $495.22 \pm 103.40^{\mathrm{pq}}$ & $485.65 \pm 101.40^{\text {qr }}$ & $490.16 \pm 102.35$ \\
\hline Average & & $386.23 \pm 80.64$ & $453.90 \pm 94.77$ & $491.40 \pm 102.60$ & $443.84 \pm 92.67$ \\
\hline \multicolumn{6}{|l|}{ Growth period ( 70 days) } \\
\hline \multirow[t]{3}{*}{ Acetic acid $(\%)^{*}$} & P1 & $78.69 \pm 3.21^{p}$ & $75.08 \pm 3.06^{\mathrm{r}}$ & $77.79 \pm 3.17 \mathrm{p}$ & $77.19 \pm 3.15$ \\
\hline & P2 & $71.72 \pm 2.93 q$ & $76.33 \pm 3.11^{\mathrm{r}}$ & $77.27 \pm 3.15 \mathrm{p}$ & $75.11 \pm 3.06$ \\
\hline & P3 & $80.03 \pm 3.27 \mathrm{p}$ & $74.93 \pm 3.06^{\mathrm{r}}$ & $70.85 \pm 2.89 q$ & $75.27 \pm 3.07$ \\
\hline Average & & $76.81 \pm 3.13$ & $75.45 \pm 3.08$ & $75.30 \pm 3.07$ & $75.85 \pm 3.09$ \\
\hline \multirow[t]{3}{*}{ Propionic acid $(\%)^{*}$} & P1 & $10.31 \pm 1.09^{\mathrm{gr}}$ & $8.11 \pm 0.85^{\mathrm{s}}$ & $9.38 \pm 0.99^{r}$ & $9.27 \pm 0.97$ \\
\hline & P2 & $14.52 \pm 1.53 \mathrm{p}$ & $9.72 \pm 1.02^{\mathrm{rs}}$ & $12.10 \pm 1.27 \mathrm{q}$ & $12.11 \pm 1.27$ \\
\hline & P3 & $9.71 \pm 1.02^{\mathrm{r}}$ & $10.42 \pm 1.10^{\mathrm{qr}}$ & $8.82 \pm 0.93^{r}$ & $9.65 \pm 1.01$ \\
\hline Average & & $11.51 \pm 1.21$ & $9.42 \pm 0.99$ & $10.10 \pm 1.06$ & $10.34 \pm 1.09$ \\
\hline \multirow[t]{3}{*}{ Butyric acid (\%) } & P1 & $16.24 \pm 4.43$ & $18.67 \pm 5.09$ & $15.14 \pm 4.13$ & $16.68 \pm 4.55$ \\
\hline & $\mathrm{P} 2$ & $14.91 \pm 4.07$ & $16.40 \pm 4.47$ & $11.71 \pm 3.19$ & $14.34 \pm 3.91$ \\
\hline & P3 & $14.21 \pm 3.87$ & $17.12 \pm 4.67$ & $22.18 \pm 6.04$ & $17.83 \pm 4.86$ \\
\hline Average & & $15.12 \pm 4.12$ & $17.40 \pm 4.74$ & $16.34 \pm 4.45$ & $16.29 \pm 4.44$ \\
\hline \multirow[t]{3}{*}{$\mathrm{TOA}(\mathrm{mmol} / \mathrm{L})^{*}$} & P1 & $147.74 \pm 31.75^{\mathrm{rs}}$ & $215.75 \pm 46.36^{\mathrm{pq}}$ & $216.40 \pm 46.51^{p q}$ & $193.30 \pm 41.54$ \\
\hline & P2 & $222.80 \pm 47.88^{p}$ & $180.46 \pm 38.78^{\text {pqrs }}$ & $156.13 \pm 33.55^{\mathrm{qrs}}$ & $186.46 \pm 40.07$ \\
\hline & P3 & $183.51 \pm 39.44^{\mathrm{pqr}}$ & $197.99 \pm 42.55^{\mathrm{pqr}}$ & $119.57 \pm 25.70^{\mathrm{s}}$ & $167.02 \pm 35.89$ \\
\hline Average & & $184.68 \pm 39.69$ & $198.07 \pm 42.56$ & $164.03 \pm 35.25$ & $182.26 \pm 39.17$ \\
\hline
\end{tabular}

Note: $\left.{ }^{*}\right)$ Means in the same combination row and column with different superscripts differ significantly $(\mathrm{p}<0.05)$.

**) Means in the same column with different superscripts differ significantly $(p<0.05)$.

F1, F2, and F3= 3 levels of NDF ratio; P1, P2, and P3= 3 sizes of fiber particles. 
of acetic acid and propionic acid contents of the caecum were $75.85 \%$ and $10.34 \%$, respectively (Table 5 ).

The difference in the size of fiber particles had a significant effect $(p<0.05)$ on the percentage of butyric acid in the contents of the caecum. The highest mean value of butyric acid percentage in the caecum contents was found in group P1 which was $19.35 \%$ with an average of all treatments of $15.11 \%$ in the post-weaning rabbits (Table 5). NDF content and fiber particle size gave a significant interaction effect $(p<0.05)$ on the total content of organic acids in the caecum contents during the post-weaning period in all treatments with an average of $443.84 \mathrm{mmol} / \mathrm{L}$ (Table 5) and during the growth period with a mean of $182.26 \mathrm{mmol} / \mathrm{L}$ (Table 5). Meanwhile, butyric acid percentage and total organic acid in the caecum contents in F1 group during the postweaning period showed the lowest average of $12.92 \%$ of the total average of $15.11 \%$ and $386.23 \mathrm{mmol} / \mathrm{L}$ of the total average of $443.84 \mathrm{mmol} / \mathrm{L}$ (Table 5).

\section{DISCUSSION}

The average temperature in the cage had a normal temperature range while the humidity was in the range of humid condition. The maximum temperature of $29^{\circ} \mathrm{C}$ is the optimal limit of comfort temperature in rabbit maintenance and temperatures below $32^{\circ} \mathrm{C}$ did not affect the digestibility value of animal feed ingredients. The decrease in insoluble fiber concentration in diets based on traditional sources of fiber led to the decrease of feed consumption and to a greater increase of ileal DM digestibility. Consequently, rabbits fed the diet with the lowest level of fiber had the highest growth rate and the lowest feed conversion among the fattening rabbits (Blas \& Wiseman, 2010; Jang et al., 2014).

\section{Production Performance}

The combination of NDF level and fiber particle size had a significant result during the fattening period of NZW cross breeding rabbits. This result is in line with the results of Gidenne et al. (2013) which stated that NDF was the main factor affecting the level of feed intake after the post-weaning period. In addition to NDF, the size and density of low fiber particles caused an increase in peristalsis to the colon so that the fibers were released through the feces quickly. This condition causes differences in the digestive pattern of rabbits and other livestock, thus affects the level of consumption (Tazzoli et al., 2013; Trocino et al., 2011, 2013).

The F2 treatment had the highest average of body weight gain and feed conversion ratio. These highest results are more related to the meeting of nutritional needs, not due to the size of the fiber particles in the feed. Cellulose is an energy source which is the main component of the NDF fraction in addition to lignin, hemicellulose, and insoluble ash content. NDF is a source of energy obtained from fiber fermentation which is mostly cellulose (Tazzoli et al., 2013). Low cellulose digestibility will cause low energy production. Lack of energy caused the rabbits tended to consume more feed but the feeds were not converted properly into the daily body weight gain and vice versa (Blas \& Wiseman, 2010).

\section{Digestibilities of Dry Matter, Crude Fiber and Fiber Fraction, Digestive Tract, and Microbial Profiles}

There was a significant interaction effect of NDF content and fiber particle size on dry matter digestibility during the post-weaning period and growth period in NZW crossbred rabbits, but this value was still within the normal range. There was a significant interaction effect of NDF concentration and fiber particle size on the digestibility of crude fiber during the growth period. This significant interaction effect was caused by the size of the fiber particles in the feed of rabbits greatly affected fiber digestibility in the digestive tract (Gidenne et al., 2013). The average digestibility of dry matter and crude fiber in rabbits was between $26 \%-76 \%$. High fiber content significantly improved the digestibilities of $\mathrm{CF}, \mathrm{EE}, \mathrm{DM}$, and $\mathrm{CP}$ which lead to improving growth performance. Dietary fiber can affect both the digestive transit and the microbial fermentative activity in rabbits. The effects of dietary fiber on digestibility are dependent on dietary fiber level and fiber digestibility and the degree of lignification being the main factor responsible for the low digestibility (Gidenne et al., 2013; Trocino et al., 2013). The size of the fiber particles affected digestion and was used for intestinal motility in rabbits (Marguenda et al., 2012). F1 showed the lowest level of NDF on crude fiber digestibility while it showed the highest crude fiber digestibility during the post-weaning period. This result was caused by the antiperistaltic drives of non-fiber components such as starch, protein, and liquid or still maintains non-fiber components which were easily digested to be fermented in the caecum. Non-digested fiber is rapidly removed through feces through peristaltic contraction to the colon (Tazzoli et al., 2013 and Trocino et al., 2011). There was a significant interaction effect of NDF treatment and the size of fiber particle on the digestibilities of NDF, ADF, and cellulose during the post-weaning and growth periods, but these values were still within the normal ranges. The ranges of NDF, ADF, cellulose, and lignin digestibilities in rabbits according to Blas \& Wiseman (2010) were $34 \%-71 \%, 23 \%-83 \%, 15 \%-59 \%$, and $8 \%-25 \%$, respectively. The digestion coefficients of CF were considerably depended on the type, source, and fractions of dietary fiber (cellulose, hemicellulose, and lignin). The increased fiber level resulted in a greater fermentation in the caecum, thus reduced caecum $\mathrm{pH}$ as a result of increasing VFA production. The increased total VFA may be attributed to the effect of the decreasing $\mathrm{pH}$ and $\mathrm{NH}_{3}-\mathrm{N}$, that eventually increased the digestible NDF, ADF, and cellulose (Gidenne et al., 2013; Martinez et al., 2013; Zku et al., 2013).

There was an effect of giving high NDF levels on lignin digestibility during the post-weaning period, especially in the F1 group. The difference in the digestibility level in this study proved that cellulose and lignin could still be digested by rabbits during the fattening period. According to Tazzoli et al. (2013), cellulose and lignin are NDF components that can still be decom- 
posed or digested, although according to Trocino et al. (2011) cellulose and lignin were fiber components that had very low digestibility in rabbits.

There was no effect of NDF level and fiber particle size on the length of the caecum and appendicitis per weight. NDF and fiber particle size affected the development of the digestive tract organs, the digestive process, and the presence of gastrointestinal microbes. Rabbits diets containing high NDF was noted to have the best feed conversion. These improvements in feed conversion and the lowest feed intake may be attributed to the effect of fiber in decreasing $\mathrm{NH}_{3}-\mathrm{N}$, pathogenic bacteria count, and increasing caecum and appendicitis length. The size and function of the digestive tract had begun to be perfect at the ages of 8 weeks to 10 weeks (De Blas et al., 2012; Gidenne et al., 2013; Volek et al., 2011). The F1P3 and F3P1 groups during the post-weaning period showed the highest mean values of caecum length per life weight whereas, during the growth period, the F1P1 and F3P2 groups showed the highest values on the average length of caecum per live weight. The digestive system during the fattening period of rabbits undergoes change or alter the adaptation to a herbivore diet. This change also occurs during the process of physical digestion (teeth), enzymatic digestion, digestion in the caecum-colic (caeco-colic) with the assist of the activity of microorganisms in the cecal appendix and the digestion of particles with the digestive system caecotrophy (Gidenne et al., 2013; De Blas et. al., 2012).

There was a significant interaction effect of the level of NDF treatment and fiber particle size on the number of E. coli bacteria in the contents of the caecum. F1 group had the highest average population of E. coli in the contents of the caecum even though during the postweaning period F1 group rabbit had the lowest average values of butyric acid percentage and total organic acid in the caecum contents. Reduction of NDF levels in feed from $300 \mathrm{~g} / \mathrm{kg}$ to $250 \mathrm{~g} / \mathrm{kg}$ caused an increase in the microflora in the ileum and decreased microflora in the caecum (Marguenda et al., 2012; Villamide et al., 2010). The increased fiber content of the feed can significantly increase VFA and reduce total lactic acid, E. coli $\mathrm{pH}$, and mortality (Knudsen et al., 2014). Rabbit in the P2 group had the highest fiber particle size which could suppress the growth of E. coli bacteria on the contents of the caecum with the highest average or the lowest contamination rate. The smaller the particle size of the fiber, the greater the ratio of surface area and volume of granules from fermented hemicellulose and cellulose to produce more VFA. The degradation of fiber for VFA production has a relationship with $\mathrm{pH}$ level (Morais et al., 2018). Increased VFA production decreases $\mathrm{pH}$ in the cecum contents which can reduce the polarization of $E$. coli and total lactic acid bacteria (anaerobic bacteria) (Trocino et al., 2010; Zku et al., 2013).

There was a significant interaction effect of NDF content and fiber particle size on the total content of lactic acid bacteria in the caecum contents both during the post-weaning period and during the growth period in NZW crossbred rabbits. It could be shown that F2P2 group had the highest population of lactic acid bacteria during the post-weaning period and the growth period. This result is in line with what was done by De Blas et al. (2012), namely when the NDF diet was reduced from 300 to $250 \mathrm{~g} / \mathrm{kg}$ the microflora of the gastrointestinal tract would change. Conversely, high fiber consumption results in infection or an increase in digestive colibacillosis reproduction (Marguenda et al., 2012). After this critical period, digestive processes can be optimized and adapted to the available feedstuffs (Gidenne et al., 2013; Jang et al., 2014).

\section{Proportional VFA and Total Organic Acid}

There was a significant interaction effect of NDF level and the size of fiber particle on the percentage of acetic and propionic acid in the caecum contents during the post-weaning period. There was a significant effect of the size of fiber particle on the percentage of butyric acid in the contents of the caecum where P1 group had the highest mean value of butyric acid percentage in the caecum contents. The results in this study showing an increase in butyric acid in the contents of the caecum are in line with the decrease in the size of fiber feed particles. The decreases in the ratio of fiber to starch and the size of fiber particles resulted in a significantly increased butyrate (Gidenne et al., 2013; Marguenda et al., 2012). The high fiber level tended to increase acetate and reduce propionate and butyrate when compared with a low dietary CF level. The concentration of the VFA in the caecum of growing rabbits was significantly increased when the fiber level was reduced and was associated with the concentration of a high molar proportion of the butyrate (Gidenne et al., 2013; Martinez et al., 2013).

There was a significant interaction effect of NDF content and fiber particle size on the total content of organic acids in the caecum contents during the postweaning period in all treatments and during the growth period. Blas \& Wiseman (2010) stated that in rabbits, the total range of organic acids was $351 \mathrm{mmol} / \mathrm{L}$, with $75 \%$ acetic acid, $17 \%$ propionic acid, and $8 \%$ butyric acid. The difference in total organic acids changes the ecosystem in the caecum and is an indication of digestive disorders. This condition was a major cause of diarrhea (due to $E$ coli), caecum disorders, mucus excretion, and low feed intake (Gidenne et al., 2013; Marguenda et al., 2012; Zku et al., 2013). It could be shown that F3P2 group had the highest total of organic acids in the caecum contents and the lowest E. coli infection during the postweaning period.

\section{CONCLUSION}

Level of NDF treatments and fiber particle size have influenced feed consumption, body weight gain, feed conversion ratio, dry matter and fiber digestibility, stress status, E. coli, total lactic acid bacteria, acetic acid, propionic acid, butyric acid, and total organic acid during the fattening period of the rabbit. The combination of F3P2 treatment was the best feed in this study, which was indicated by a high feed consumption, high body 
weight gain, low feed conversion ratio, the lowest stress status, the lowest E. coli, and the highest total organic acid in rabbits during the fattening period.

\section{CONFLICT OF INTEREST}

We certify that there is no conflict of interest with any financial, personal, or other relationships with other people or organization related to the material discussed in the manuscript.

\section{ACKNOWLEDGEMENT}

Thank you, the author, to CV. Tlogowungu Farm Pati Central Java for all the facilities provided, all funding, and the place until this research can be completed according to expectations.

\section{REFERENCES}

Abad, R., M.A. Ibanez, R. Carabano, \& J. García. 2013. Quantification of soluble fiber in feedstuffs for rabbits and evaluation of the interference between the determinations of soluble fiber and intestinal mucin. Anim. Feed Sci. Technol. 182: 61-70. https://doi.org/10.1016/j. anifeedsci.2013.04.001

AOAC. 2012. Official Methods of Analysis. $18^{\text {th }}$ Ed. Association of Official Analytical Chemist, Mayland, USA.

Blas, C. \& J. Wiseman. 2010. Nutrition of The Rabbit. $2^{\text {nd }}$ Edition. CU Madrid, Spain and Nottingham UK.

De Blas, J.C., S. Chamorro, J.G. Alonso, P.G. Rebollar, A.I.G.Ruiz, M.S.G. Conde, D. Menoyo, N. Nicodemus, C. Romero, \& R. Carabano. 2012. Nutritional digestive disturbances in weaner rabbits. Anim. Feed Sci. Technol. 173: 102-11. https://doi.org/10.1016/j.anifeedsci.2011.12.016

Directorate General of Livestock and Animal Health, Ministry of Agriculture, Republic of Indonesia. 2017. Statistics on Animal Husbandry and Animal Health. http://ditjenpkh. pertanian.go.id/userfiles/File/Buku_Statistik_ 2017_ (ebook). pdf?time $=1505127443012$

Gidenne, T., V. Kerdiles, N. Jehl, P. Arveux, B. Eckenfelder, C. Briens, S. Montessuy, \& G. Muraz. 2013. Protein replacement by digestible fibre in the diet of growing rabbits. Anim. Feed Sci. Technol. 183: 142-150. https://doi. org/10.1016/j.anifeedsci.2013.03.013

Jang, Y.D., S.K. Jang, D.H. Kim, H.K. Oh, \& Y.Y. Kim. 2014. Effects of dietary protein levels for gestating gilts on reproductive performance, blood metabolites and milk composition. Asian Australas. J. Anim Sci. 27: 83-92. https://doi. org/10.5713/ajas.2013.13369

Knudsen, C., S. Combes, C. Briens, G. Coutelet, J. Duperray, Salaun, A. Travel, D. Weissman, \& T. Gidenne. 2014. Increasing the digestible energy intake under a restriction strategy improves the feed conversion ratio of the growing rabbit without negatively impacting the health status. Livest Sci. 169: 96-105. https://doi.org/10.1016/j. livsci.2014.08.015
Marguenda, I., N. Nicodemus, S. Vadillo, L. Sevilla, M. Villarroel, C. Romeo, \& R. Carabaño . 2012. Effect of dietary type and level of fiber on rabbit carcass yield and its microbiological characteristics. Livest. Sci. 145: 7-12. https://doi.org/10.1016/j.livsci.2011.12.012

Martínez, V.B., P.E. Martínez, L. Rodenas,V.J. Moya, C. Cervera, J.J. Pascual, \& E. Blas. 2013. Partial replacement of starch with acid detergent fibre and/or neutral detergent soluble fibre at two protein levels: effects on ileal apparent digestibility and caecal environment of growing rabbits. Livest. Sci. 154: 123-130. https://doi.org/10.1016/j. livsci.2013.02.012

Morais, M. J., C. C. Sevilla, J E. E. C. Abes, \& A. A. Angeles. 2018. Growth performance and ruminal metabolic variables of goats fed rain tree (Samanea saman). Trop. Anim. Sci. J. 41:22-28. https://doi.org/10.5398/tasj.2018.41.1.22

Sobri, M., K.G. Wiryawan, P.D.M.H. Karti, \& W.T. Wibawan. 2019. Effect of fiber feed on rabbits during the postweaning period. Pak. J. Nutr. 18: 101-108. https://doi.org/10.3923/ pjn.2019.101.108

Steel, R. G. D. \& J. H. Torrie. 1995. Principles and Procedures of Statistics. Alih Bahasa Sumantri, B. Prinsip dan Prosedur Statistika. Edisi 4 Penerbit PT. Gramedia Pustaka Utama, Jakarta.

Tazzoli, M., M. Birolo, E. Filiou, A. Zuffelato, \& G. Xiccato 2013. Increasing dietary energy with starch and soluble fibre and reducing ADF at different protein levels for growing rabbits. Agriculturae Conspectus Scientificus. 78: 1-5.

Trocino, A., J. Garcia, R. Carabano, \& G. Xiccato. 2013. A metaanalysis of the role of soluble fibre in diets for growing rabbits. World Rabbit Science 21:1-15. https://doi.org/10.4995/ wrs.2013.1285

Trocino A., M. Fragkiadakis, \& G. Xiccato. 2011. Effect ofthe increase of dietary starch and soluble fibre on digestive efficiency and growth performance of meat rabbits. Anim. Feed Sci. Technol. 165: 265-277. https://doi.org/10.1016/j. anifeedsci.2011.03.008

Trocino, A., M. Fragkiadakis, G. Radaelli, \& G. Xiccato. 2010. Effect of dietarysoluble fibre level and protein source on growth, digestion, caecal activity and health of fattening rabbits. World Rabbit Science 18: 199-210. https://doi. org/10.4995/wrs.2010.779

Van Soest, P.J., J.B. Robertson, \& B.A. Lewis. 1995. Methods for dietary fiber, neutral detergent fiber and on-starch polysaccharides in relation to animal nutrition. J. Dairy Sci. 74: 3583-3597. https://doi.org/10.3168/jds. S0022-0302(91)78551-2

Villamide, M.J., R. Carabano, L. Maertens, J. Pascual, T. Gidenne, L.F.E. Cunha \& G. Xiccato. 2010. Prediction of the nutritional value of European compound feeds for rabbits by chemical components and in vitro analysis. Anim. Feed Sci. Technol. 150: 283-294. https://doi.org/10.1016/j. anifeedsci.2008.09.007

Volek, Z. \& M. Marounek. 2011. Dried chicory root (Cichorium intybusL.) as anatural fructan source in rabbit diet: effects on growth performance, digestion and caecal and carcass traits. World Rabbit Science 19: 143-150. https://doi. org/10.4995/wrs.2011.850

Zhu, Y.L., C.Y. Wang, X.P. Wang, B. Li, L.Z. Sun, \& F.C. Li. 2013. Effects of dietary fiber and starch levels on the nonspecific immune response of growing rabbits. Livest Sci. 155: 285-93. https://doi.org/10.1016/j.livsci.2013.04.018 